\title{
The PLC Signals' Noise Mitigating Algorithm with PCA
}

\author{
MingYue Zhai \\ School of Computer and Information Engineering \\ Guangdong University Of Petrochemical Technology, Maoming, China
}

Keyword: Smart grids; PLC; PCA; Noise mitigation

\begin{abstract}
Noises in the PLCPLC) systems are too severe. In the paper, we proposed a new method to mitigate such noises based on the principal components analyses(PCA). There are 4 steps in this algorithm. Signals generalization is the the first step. the one dimension PLC signal is extended to a multiple dimensions signals. In the second step, the new signals are decorrelated by adding the white noises. For such the uncorrelated data set in the transformed domain, we use the PCA technology to mitigate noises. The useful PLC signals can be recovered with applying the inverse transform matrix as the forth step. We verify this algorithm by the simulations.
\end{abstract}

\section{Introduction}

In China, a significant amount of pollution gas emissions was produce by the conventional power systems[1-7]. Ti reduce such emissions, smart grids are proposed. The essential part of smart grid is a communication infrastructure with two-way communications. One of the promising technologies is the Power line communication (PLC). The PLC could be used in smart grids.

Principal component analysis (PCA) is considered as one of the most valuable applied linear algebra's results. A path is provided with the PCA to transform a complex data set to a lower dimension. With such transformation, the hidden information can be revealed and thus we can obtain the dynamics on the simplified construction. Therefore, it is often used in data set analyses, not in signal processing. Until now, there is no paper to discuss the application of PCA to noise reduction in seismic data processing.

In the paper, random noises are mitigated by the proposed new algorithm with the concept of PCA . In this method, the received PLC signals are transformed to a linear of signal components, spanned bythe received signal's covariance matrix's eigenvectors. In such domain, signals of interesting are with greater powers than random noises, and powers of random noises, therefore, can be forced to be zero. By doing so, random noises can be mitigated greatly in the time domain. At the same time, we evaluate the performance of the noise mitigation scheme based on the PCA technology in seismic signal processing applications.

\section{Fundamentals of the Principal Components Analyses}

In the PLC systems, the signals can be expressed as a matrix with $m \times n$ dimensions, where the $\mathrm{n}$ columns are the samples (e.g. observations) and the $m$ rows are the variables. We wish to linearly transform this matrix, $\mathbf{X}$ into another matrix, $\mathbf{Y}$, also of dimension $m \times n$ so that for some $m \times m$, $\mathbf{P}$,

$\mathbf{Y}=\mathbf{P X}$

This equation represents a change of basis. If we rewrite $\mathbf{P}$ as $\mathbf{P}^{T}=\left[\mathbf{p}_{1}^{T}, \mathbf{p}_{2}^{T}, \cdots, \mathbf{p}_{m}^{T}\right]$, where $\mathbf{p}_{i}^{T}$ is the $i^{\text {th }}$ row vector of $\mathbf{P}$, and $\mathbf{X}$ as $\mathbf{X}=\left[\mathbf{x}_{1}, \mathbf{x}_{2}, \cdots, \mathbf{x}_{n}\right]$, then Eq.1 can be interpreted in the following way, 


$$
\mathbf{Y}=\mathbf{P X}=\left[\begin{array}{c}
\mathbf{p}_{1} \\
\mathbf{p}_{2} \\
\vdots \\
\mathbf{p}_{m}
\end{array}\right]\left[\begin{array}{llll}
\mathbf{x}_{1} & \mathbf{x}_{2} & \cdots & \mathbf{p}_{m}
\end{array}\right]
$$

and it can be expressed further as

$$
\mathbf{Y}=\mathbf{P X}=\left[\begin{array}{cccc}
\mathbf{p}_{1} \mathbf{x}_{1} & \mathbf{p}_{1} \mathbf{x}_{2} & \cdots & \mathbf{p}_{1} \mathbf{x}_{n} \\
\mathbf{p}_{2} \mathbf{x}_{1} & \mathbf{p}_{2} \mathbf{x}_{2} & \cdots & \mathbf{p}_{2} \mathbf{x}_{n} \\
\vdots & \vdots & \ddots & \vdots \\
\mathbf{p}_{m} \mathbf{x}_{1} & \mathbf{p}_{m} \mathbf{x}_{2} & \cdots & \mathbf{p}_{m} \mathbf{x}_{n}
\end{array}\right]
$$

We can note the form of each column of $\mathbf{Y}, \mathbf{y}_{i}$ as

$$
\mathbf{y}_{i}=\left[\begin{array}{c}
\mathbf{p}_{1} \mathbf{x}_{i} \\
\mathbf{p}_{2} \mathbf{x}_{i} \\
\vdots \\
\mathbf{p}_{m} \mathbf{x}_{i}
\end{array}\right]
$$

We found that the coefficient of $\mathbf{y}_{i}$ is a dot product of $\mathbf{x}_{i}$ with the corresponding row in $\mathbf{P}$. In other words, $\mathbf{p}_{i} \mathbf{x}_{j}$ is just the standard Euclidean inner (dot) product for $\mathbf{p}_{i}, \mathbf{x}_{j} \in \mathbf{R}^{m}$. This tells us that the original data, $\mathbf{X}$ is being projected on to the rows of $\mathbf{P}$. Thus, the rows of $\mathbf{P}$, $\left\{\mathbf{p}_{1}, \mathbf{p}_{2}, \cdots, \mathbf{p}_{m}\right\}$ are a new basis for representing the columns of $\mathbf{X}$.

Until now, there is no rule to define the transformation matrix $\mathbf{P}$, however, the PCA method use some tricks. In such method, the matrix $\mathbf{P}$ is selected to be a matrix where each row $\mathbf{p}_{i}$ is an eigenvector of $\mathbf{X X}^{T}$. For a positive definite or positive semi-definite matrix, like covariance matrix $\mathbf{X} \mathbf{X}^{T}$, we have the following results:

$$
\mathbf{S}_{\mathbf{X}}=\mathbf{X} \mathbf{X}^{T}=\mathbf{E D E}^{T}
$$

where $\mathbf{E}$ is an $m \times m$ orthogonal matrix whose columns are the orthogonal eigenvectors of $\mathbf{X X}^{T}$, and $\mathbf{D}$ is a diagonal matrix which has the eigenvalues of $\mathbf{X} \mathbf{X}^{T}$ as its (diagonal) entries.

Therefore, by the mentioned selection,

$$
\mathbf{P}=\mathbf{E}^{T}
$$

Substituting Eq.6 into the covariance matrix $\mathbf{S}_{\mathbf{Y}}=\mathbf{Y} \mathbf{Y}^{T}$, we have

$$
\mathbf{S}_{\mathbf{Y}}=[\mathbf{P X}][\mathbf{P X}]^{T}=\mathbf{P X X} \mathbf{X}^{T} \mathbf{P}^{T}=\mathbf{E}^{T}\left[\mathbf{E D E}^{T}\right] \mathbf{E}=\mathbf{D}
$$

For Eq.7, there is a important assumption in the PCA method. The new variables are in the new basis space $\left\{\mathbf{p}_{1}, \mathbf{p}_{2}, \cdots, \mathbf{p}_{m}\right\}$. Ath the same times, these new variables are as uncorrelated as possible, but with powers as large as possible. It means that covariances of different variables in the covariance matrix $\mathbf{S}_{\mathbf{Y}}$, should be as close to zero as possible, with variances as large as possible.

\section{The Proposed Noise Mitigation Based on PCA}

From the definitions of PCA, we can see that the PCA can get the principal components of the data $\mathbf{X}$, corresponding to the eigenvalues of the matrix $\mathbf{X} \mathbf{X}^{T}$. Thus, its most common use is as the first step in trying to analyze large data sets, such as dimension reduction, blind source separation, and data compression. When applying it to de-noising, the used data are often graphics, that is to see the data is with two dimensions at least. Until now, there is no paper to discuss the application of PCA to seismic signals de-noising. The reason is that signals with one dimension, such as 
seismic signal $x(t)$, is without eigenvectors and thus the transform matrix $\mathbf{P}$ can't be formalized according to Eq.efEq.transform.

In the paper, we proposed a new scheme to mitigate random noises based on PCA in noisy seismic signals. Suppose the noisy seismic signal $y_{0}(t)$ as:

$$
y_{0}(t)=x(t)+n_{0}(t)
$$

where $x(t)$ is the seismic signal of interesting, and $n_{0}(t)$ is random noise at time $t$. The discrete versions of such signal are as follows with sample interval $\Delta$ :

$$
y_{0}(k)=x(k)+n_{0}(k) \quad k=0,1, \cdots, K-1
$$

where $K$ is the time length of signal in discrete version. Therefore, the discrete seismic signal can be expressed in the form of column vector:

$$
\mathbf{y}_{0}=\left[\begin{array}{c}
y_{0}(0) \\
y_{0}(1) \\
\vdots \\
y_{0}(K-1)
\end{array}\right]=\left[\begin{array}{c}
x(0)+n_{0}(0) \\
x(1)+n_{0}(1) \\
\vdots \\
x(K-1)+n_{0}(K-1)
\end{array}\right]
$$

In order to use PCA, we generalized the column vector $\mathbf{y}_{0}$ to a new data matrix $\mathbf{Y}$ first:

$$
\mathbf{Y}=\left[\begin{array}{llll}
\mathbf{y}_{0} & \mathbf{y}_{1} & \cdots & \mathbf{y}_{L-1}
\end{array}\right]
$$

where $L$ is the number of the generalization, and can also be called signal generalization dimension $D_{\text {generalization }}$ in the paper. For the $l^{\text {th }}$ generalized noisy signal $\mathbf{y}_{l}$, it can be expressed as:

$$
\begin{aligned}
\mathbf{y}_{l} & =\left[\begin{array}{c}
y_{l}(0) \\
y_{l}(1) \\
\vdots \\
y_{l}(K-1)
\end{array}\right] \\
& =\left[\begin{array}{c}
x(0)+n_{l}(0)+n_{0}(0) \\
x(1)+n_{l}(1)+n_{0}(1) \\
\vdots \\
x(K-1)+n_{l}(K-1)+n_{0}(K-1)
\end{array}\right]
\end{aligned}
$$

where $n_{l}(k), k=0,1, \cdots, K-1$ is the random noise at $t=(k-1) \Delta$ in the $l^{\text {th }}$ generalized noisy signal $\mathbf{y}_{l}$. Because $n_{l}(k), k=0,1, \cdots, K-1$ is a random process, it has the following properties:

$$
\begin{gathered}
E\left[n_{l}(k)\right]=0 \\
E\left[n_{l}(k) n_{j}(q)\right]=\left\{\begin{aligned}
\sigma_{l}^{2}, \text { for } & j=l \text { and } q=k \\
0, & \text { else }
\end{aligned}\right.
\end{gathered}
$$

where $E[\bullet]$ is mathematical expectation operator, and $\sigma_{l}^{2}$ is the variance(or power) of the random noise $n_{l}(k)$. This step is called signal whitening in the paper. Signal whitening can represent the original signal $y_{0}(k)$ with one dimension as a data set $\mathbf{Y}$, where all of the new signals(corresponding to column vectors) are uncorrelated with the original signal $y_{0}(k)$. Thus, the $l^{\text {th }}$ column of the data set $\mathbf{Y}$ is with the seismic signal of interesting $x(k)$ and the random noise $n(k)=n_{0}(k)+n_{l}(k)$.

In the next step, PCA is applied to data set $\mathbf{Y}$, and we have

$$
\mathbf{Z}=\mathbf{P Y}
$$




$$
\mathbf{P}=\left[\begin{array}{c}
\mathbf{p}_{1} \\
\mathbf{p}_{2} \\
\vdots \\
\mathbf{p}_{K}
\end{array}\right]
$$

where, each row vector $\mathbf{p}_{k}$ is the $k^{\text {th }}$ eigenvector of $\mathbf{Y Y} \mathbf{Y}^{\mathbf{T}}$. At the same time, $\mathbf{p}_{k}$ is placed in descending order of the corresponding eigenvalues of $\mathbf{Y Y} \mathbf{Y}^{\text {athbfT }}$. Thus, $\mathbf{p}_{1}$ is corresponding to the largest eigenvalue of $\mathbf{Y Y}^{\mathbf{T}}$.

In the third step, we select the previous $D_{\text {reco }}$ row vectors of transform matrix $\mathbf{P}$ to formalize a new transform matrix $\mathbf{P}_{\text {reco }}$ :

$$
\mathbf{P}_{\text {reco }}=\left[\begin{array}{c}
\mathbf{p}_{1} \\
\mathbf{p}_{2} \\
\vdots \\
\mathbf{p}_{D_{\text {reco }}}
\end{array}\right]
$$

In this matrix, the row vectors correspond to $D_{\text {reco }}$ largest eigenvalues in the original matrix $\mathbf{P}$. Such $D_{\text {reco }}$ largest eigenvalues are considered as the powers of the generalized signal of interesting, $x(k), k=0,1, \cdots, K-1$. After getting transforming matrix $\mathbf{P}_{\text {reco }}$, we have

$$
\mathbf{Z}_{\text {reco }}=\mathbf{P}_{\text {reco }} \mathbf{Y}
$$

In order to get the seismic signals of interesting, we apply the inverse matrix of $\mathbf{P}_{\text {reco }}$ to $\mathbf{Z}_{\text {reco }}$ :

$$
\mathbf{X}=\mathbf{P}_{\text {reco }}^{T} \mathbf{Z}_{\text {reco }}
$$

\section{Applications of the Proposed Method}

Strong inferences can be found in received OFDM signals, and such system with noises can be modeled as the Doppler signals. Separation of the noises and the signals are becoming a very difficult task only in time domain or in frequency domain.

In the paper, a new method is proposed to separate the OFDM signals and the noises with the concept of PCA. The bit-error-rate (BER) concept is used to evaluate the performance of the new algorithm. We use this new method for the simulation signals and the real measurements. For simulations data, the used block diagram of the OFDM system is shown in Fig. 1, and the simulated OFDM signal $x(t)$ can be expressed as follows:

$$
x(t)=\sum_{l=0}^{\infty} \sum_{k=0}^{N-1} X_{l}[k] e^{j 2 \pi f_{k}\left(t-l T_{s y m}\right)} .
$$

where $T_{s y m}$ is the $l^{\text {th }}$ OFDM symbol $X_{l}$ duration, and $N$ is the number of the sub-carrier in OFDM system with sub-carrier frequency $f_{k}=\frac{k}{T_{s s m}}$.

The IEEE P1901.2 standard is used to simulate the PLC signals. The frequency range is from $35.9-487.5 \mathrm{kHz}$, and the sampling frequency is set to $1.2 \mathrm{MHz}$. there are 256 sub-carriers to transmit the data. Thirty bits are inserted into the OFDM symbol as cyclic prefix with the duration of $25 \mu s$, while 22 bits as the effective cyclic prefix with the duration of $18.3 \mu \mathrm{s}$. The sub-carrier spacing is $4.6875 \mathrm{kHz}$, therefore, the OFDM symbol duration is $237.1 \mu \mathrm{s}$. QAM modulation is selected with constellation size 16 .

Fig. 1 shows the BER performance. There is a great performance improvement because of the noise mitigation. The influences by the heavy noises are reduced significantly. When the SNRs are low, there are 1 2 orders BER improvement. When the SNRs are very high, there will be 3 orders BER improvement. For example, if the SNR is about $8 d B$, the BERs are about $10^{-2}$ and 
$10^{-5}$ for without interference mitigation and with mitigation, respectively. If the SNR is about $18 d B$, the BERs are about $10^{-4}$ and $10^{-6}$, respectively.

We also applied the proposed scheme to the measurements data. The BER is improved from about $10^{-3}$ to about $10^{-5}$. The improvement is significantly.

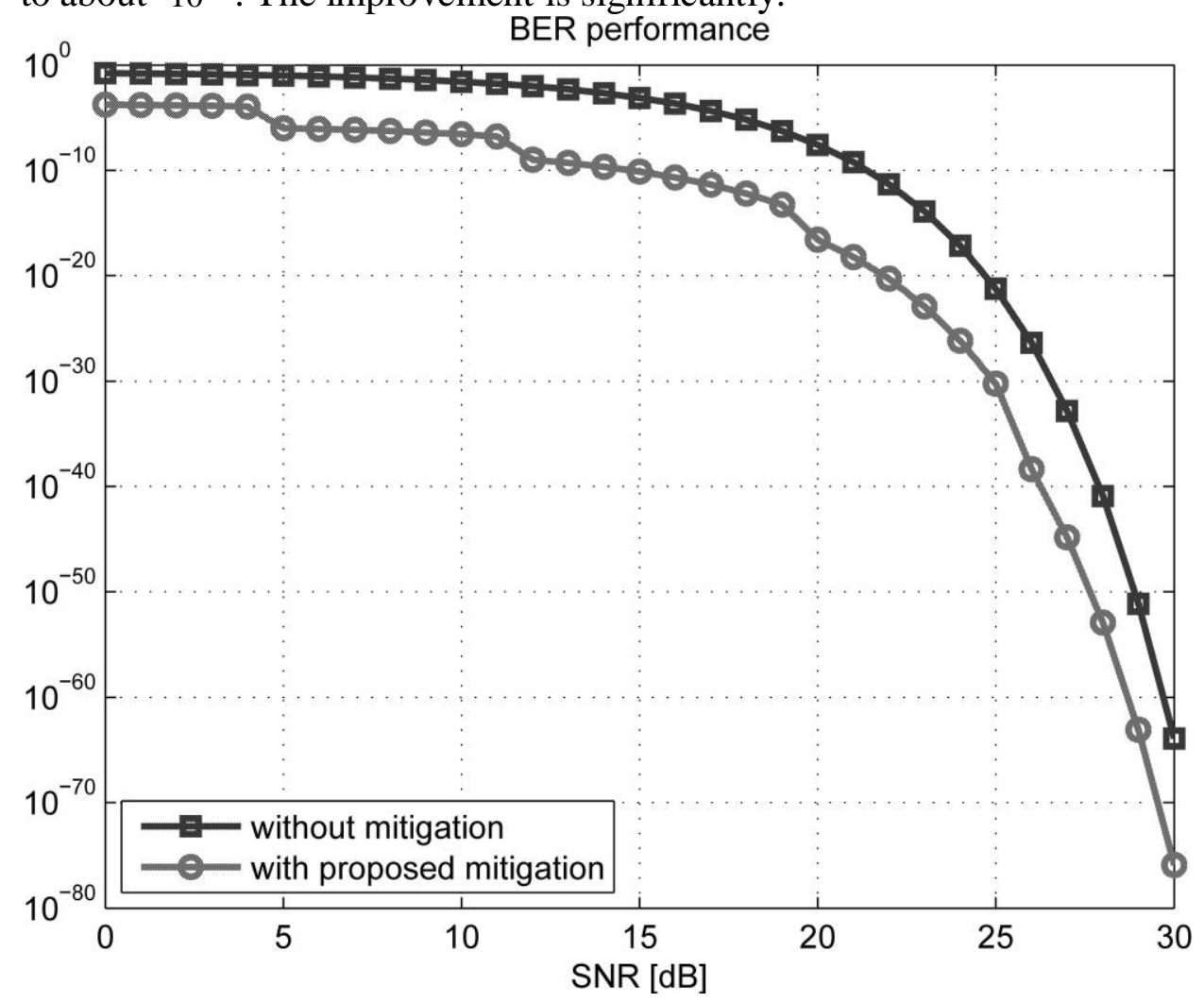

Figure 1. BER PERFORMANCES FOR THE PROPOSED METHOD

\section{Conclusions}

The heavy random noises are founded in the PLC channels, and such noises are with the overlapping frequencies because the PLC signals are also modulated on such frequencies. The PLC systems can not work well when the inferences are strong. In order to mitigate such inferences, we introduced the PCA transform to the OFDM signals processing. There are 4 steps in this algorithm. Signals generalization is the the first step. the one dimension PLC signal is extended to a multiple dimensions signals. In the second step, the new signals are decorrelated by adding the white noises. For such the uncorrelated data set in the transformed domain, we use the PCA technology to mitigate noises. The useful PLC signals can be recovered with applying the inverse transform matrix as the forth step. We verify this algorithm by the simulations.

\section{References}

[1] M. Nassar, J. Lin, Y. Mortazavi, A. Dabak, I. H. Kim, and B. L. Evans. Local utility power line communications in the $3-500 \mathrm{kHz}$ band: Channel impairments, noise, and standards. IEEE Signal Processing Magazine, 29(5):116-127, 2012.

[2] W. Stefanutti, S. Saggini, P. Mattavelli, and M. Ghioni.Power line communication in digitally controlled dc -dc converters using switching frequency modulation. Industrial Electronics, IEEE Transactions on, 55(4):1509-1518, April 2008.

[3] Zhiqiang Xu, Mingyue Zhai, and Jun Lu.Crosslayer optimization of user scheduling and resource allocation in power-line communication systems. IEEE Transactions on Power Delivery, 26(3):1449-1458, 2011.

[4] Zhiqiang Xu, Mingyue Zhai, and Yuming Zhao. Optimal resource allocation based on resource 
factor for power-line communication systems. IEEE Transactions on Power Delivery, 25(2):657-666, 2010.

[5] Sung-Guk Yoon, Seowoo Jang, Yong-Hwa Kim, and Saewoong Bahk. Opportunistic routing for smart grid with power line communication access networks. IEEE Transactions on Smart Grid, 5(1):303-311, 2014.

[6] Ming-Yue Zhai. Signal recovery in power-line communications systems based on the fractals. IEEE Transactions on Power Delivery, 26(3):1864-1872, 2011.

[7] Ming-Yue Zhai. Transmission characteristics of low-voltage distribution networks in china under the smart grids environment. IEEE Transactions on Power Delivery, 26(1):173-180, 2011.

[8] Ming-Yue Zhai. Mitigation of the moving radio interferences in the ofdm-based power line communication channels in china. International Journal of Communication Systems, pages n/an/a, 2014.

[9] Ming-Yue Zhai and Ling-Dong Su. Research on the memory order of the power-line communication channel. International Journal of Communication Systems, pages n/a-n/a, 2014. 